\title{
PARAMETRIZATIONS OF SOME TEICHMÜLLER SPACES BY TRACE FUNCTIONS
}

\author{
GOU NAKAMURA AND TOSHIHIRO NAKANISHI
}

\begin{abstract}
We show a tuple of trace functions which give a global parametrization of the Teichmüller space $\mathcal{T}(g, m)$ of types $(1,2)$ and $(2,0)$. We also show that the mapping class group acting on these Teichmüller spaces can be represented by a group of rational transformations in seven variables.
\end{abstract}

\section{INTRODUCTION}

Let $\bar{S}$ be a surface of type $(g, m)$, that is, a smooth compact and oriented surface of genus $g$ with $m$ boundary curves, and $S$ the interior of $\bar{S}$. Throughout this paper we assume that $2 g-2+m>0$. The Teichmüller space $\mathcal{T}(g, m)$ of type $(g, m)$ is the space of equivalence classes of marked complete hyperbolic metrics of curvature -1 on $S$ such that each boundary curve is homotopic to a (unique) closed geodesic curve in $S$. It is known that $\mathcal{T}(g, m)$ is a real analytic manifold homeomorphic to $\mathbb{R}^{6 g-6+3 m}$ (see, for example, [18, Section 34]).

The free homotopy class of a homotopically non-trivial closed curve $c$ in $S$ defines a real analytic function $\ell_{c}$ on $\mathcal{T}(g, m)$, called the geodesic length function, which assigns to each point in $\mathcal{T}(g, m)$ the length of the geodesic curve freely homotopic to $c$ on a marked hyperbolic surface representing the point. It is known that there are finitely many closed curves $c_{1}, \ldots, c_{N}$ on $S$ such that each point of $\mathcal{T}(g, m)$ is recovered by the values of their geodesic length functions. In other words,

$$
\left(\ell_{c_{1}}, \ldots, \ell_{c_{N}}\right): \mathcal{T}(g, m) \rightarrow \mathbb{R}_{>0}^{N} \quad\left(\mathbb{R}_{>0}=\{x \in \mathbb{R}: x>0\}\right)
$$

is an embedding (see, for example, 4, 4, 13, 14 and [15]). Then the problem of finding the minimal number $N(g, m)$ of geodesic length functions needed to parametrize $\mathcal{T}(g, m)$ globally arises naturally. This problem has been solved and

$$
N(g, m)= \begin{cases}6 g-6+3 m & \text { if } m>0 \\ 6 g-5 & \text { if } m=0 .\end{cases}
$$

For the case of $(g, 0)$, the problem was solved first by Schmutz 12. Later Okumura [10] gave a solution by a different method. The fact that $N(g, 0)>\operatorname{dim} \mathcal{T}(g, 0)$ is a consequence of Wolpert's studies (see [17) on geodesic length functions. Later in [6], Feng Luo gave a clear account of the parametrization of the Teichmüller

Received by the editors June 16, 2011.

2010 Mathematics Subject Classification. Primary 32G15; Secondary 30F35.

Key words and phrases. Teichmüller space, Fuchsian groups.

The first author was partially supported by Grant-in-Aid for Young Scientists (B) (No. 20740081), Japan Society for the Promotion of Science.

The second author was partially supported by Grand-in-Aid for Scientific Research (No. 18540179), Ministry of Education, Science and Culture of Japan. 
spaces by this minimal number of geodesic length functions. A new proof of (1.1) is contained in a paper by Hamenstädt [3].

In this paper we treat trace functions on $\mathcal{T}(g, m)$ regarded as a deformation space of Fuchsian groups. Trace functions and geodesic length functions are equivalent (see (2.2) below). We establish a trace identity (3.11) for groups of type $(1,2)$ and give seven trace functions which parametrize Teichmüller spaces of types $(1,2)$ or $(2,0)$. We also show that they allow rational representations of the mapping class groups acting on these Teichmüller spaces.

\section{Preliminaries}

2.1. Teichmüller space. Our basic references for notation and terminologies about Fuchsian groups and Teichmüller spaces are [1, [16] and [18. The group of orientation-preserving isometries of the hyperbolic plane $\mathbb{H}=\{z=x+i y: y>0\}$ is identified with $P S L(2, \mathbb{R})$. A Fuchsian group $G$ is a discrete subgroup of $P S L(2, \mathbb{R})$. The action of $G$ is naturally extended to that on the boundary $\overline{\mathbb{R}}$ of $\mathbb{H}$ in the Riemann sphere. Let $\Omega(G)$ be the maximal subset of $\overline{\mathbb{R}}$ on which $G$ acts discontinuously. The group $G$ is said to be of type $(g, m)$ if $G$ is purely hyperbolic and the factor surface $\mathbb{H} \cup \Omega(G) / G$ is a surface of type $(g, m)$. If $G$ is of type $(g, m)$, it has a canonical generator system

$$
\mathcal{S}=\left(A_{1}, B_{1}, \ldots, A_{g}, B_{g}, C_{1}, \ldots, C_{m}\right) \text { with }\left(\prod_{j=1}^{g} A_{j} B_{j} A_{j}^{-1} B_{j}^{-1}\right) C_{1} \cdots C_{m}=1
$$

where $\left(A_{1}, B_{1}\right), \ldots,\left(A_{g}, B_{g}\right)$ correspond with the handles and $C_{1}, \ldots, C_{m}$ the boundary curves of $\mathbb{H} \cup \Omega(G) / G$. We shall call $\mathcal{S}$ briefly a generator system of type $(g, m)$. Two generator systems of type $(g, m)$ are said to be equivalent if and only if they are in the same simultaneous conjugacy class. The Teichmüller space $\mathcal{T}(g, m)$ of Fuchsian groups of type $(g, m)$ is the space of equivalence classes of generator systems of type $(g, m)$. We represent a point of $\mathcal{T}(g, m)$ by $(G, \mathcal{S})$ a pair of a Fuchsian group $G$ and a canonical generator system $\mathcal{S}$ of $G$.

Let $G$ be a Fuchsian group. For a hyperbolic element $A$ of $G$, let $p_{A}$ and $q_{A}$ denote the fixed points of $A$. The axis $L_{A}$ of $A$ is the hyperbolic line between $p_{A}$ and $q_{A}$. The cyclic group $\langle A\rangle$ generated by $A$ acts on $L_{A}$, and $L_{A} /\langle A\rangle$ is a closed geodesic curve $c_{A}$ on $\mathbb{H} / G$. The length $\ell(A)=\ell_{c_{A}}$ of $c_{A}$ (counting multiplicity) satisfies

$$
|\operatorname{tr} A|=2 \cosh \frac{\ell(A)}{2}
$$

2.2. Trace identities for $S L(2, \mathbb{R})$. We show some trace identities in $S L(2, \mathbb{R})$ (see, for example, [7, §3.4]). Trace is similarity-invariant: $\operatorname{tr} P^{-1} A P=\operatorname{tr} A$ for $A$ and $P \in S L(2, \mathbb{R})$. Matrices in $S L(2, \mathbb{R})$ satisfy:

(I1) $\operatorname{tr} A=\operatorname{tr} A^{-1}$,

(I2) $\operatorname{tr} A B+\operatorname{tr} A B^{-1}=\operatorname{tr} A \operatorname{tr} B$,

(I3) $\operatorname{tr} A B C=\operatorname{tr} A \operatorname{tr} B C+\operatorname{tr} B \operatorname{tr} C A+\operatorname{tr} C \operatorname{tr} A B-\operatorname{tr} A \operatorname{tr} B \operatorname{tr} C-\operatorname{tr} A C B$.

Let $[A, B]=A B A^{-1} B^{-1}$ be the commutator of $A$ and $B$. Substituting $A^{-1} B^{-1}$ to $C$ in (I3) yields

(I4) $\operatorname{tr}[A, B]=(\operatorname{tr} A)^{2}+(\operatorname{tr} B)^{2}+(\operatorname{tr} A B)^{2}-\operatorname{tr} A \operatorname{tr} B \operatorname{tr} A B-2$. 
Let $A, B, C$ and $D \in S L(2, \mathbb{R})$ satisfy $A B C D=1$ (the identity matrix) and let $a=-\operatorname{tr} A, b=-\operatorname{tr} B, c=-\operatorname{tr} C, d=-\operatorname{tr} D, x=-\operatorname{tr} B C, y=-\operatorname{tr} C A$ and $z=-\operatorname{tr} A B$. Then

(I5)

$x^{2}+y^{2}+z^{2}-x y z+(a d+b c) x+(b d+c a) y+(c d+a b) z+a^{2}+b^{2}+c^{2}+d^{2}+a b c d-4=0$.

Given a system $\mathcal{S}=\left(A_{1}, \ldots, A_{n}\right)$ of matrices in $S L(2, \mathbb{R})$, let $G_{\mathcal{S}}=\left\langle A_{1}, \ldots, A_{n}\right\rangle$ denote the group generated by $A_{1}, \ldots, A_{n}$. We define

$$
T_{\mathcal{S}}=\left\{\operatorname{tr}\left(A_{j_{1}} A_{j_{2}} \cdots A_{j_{r}}\right): 1 \leq j_{1}<j_{2}<\cdots<j_{r} \leq n, 1 \leq r \leq n\right\} .
$$

Then, we have the following lemma.

Lemma 2.1 (see [7, §3.5]). If $g \in G_{\mathcal{S}}$, then $\operatorname{tr} g$ is a polynomial with variables in $T_{\mathcal{S}}$ and integer coefficients.

\subsection{Parametrization of the Teichmüller spaces.}

2.3.1. Trace functions. We fix a point $\left(G_{0}, \mathcal{S}_{0}\right)$ of the Teichmüller space $\mathcal{T}(g, m)$. Here we assume that elements in $\mathcal{S}_{0}$ are matrices in $S L(2, \mathbb{R})[5]$. A point $X=$ $(G, \mathcal{S})$ of $\mathcal{T}(g, m)$ is identified with the conjugacy class of the group isomorphism $\rho_{X}: G_{0} \rightarrow G$ which sends an element of $\mathcal{S}_{0}$ to its corresponding element of $\mathcal{S}$. For each $g \in G_{0}$, the trace function $\chi_{g}(X)=\operatorname{tr} \rho_{X}(g)$ is a well-defined function on $\mathcal{T}(g, m)$. We can assume that $\chi_{g}$ is continuous, because $\chi_{g}$ takes a value in $\{x \in \mathbb{R}:|x|>2\}$ and $\mathcal{T}(g, m)$ is simply connected. Then $\left|\chi_{g}\right|=\chi_{g}$ or $\left|\chi_{g}\right|=-\chi_{g}$ all over $\mathcal{T}(g, m)$. Thus, due to (2.2) we can replace geodesic length functions by trace functions. We rephrase the theorem in the introduction.

Theorem 2.1. There exist a finite number of elements $g_{1}, \ldots, g_{N}$ in $G_{0}$ such that

$$
\left(\chi_{g_{1}}, \ldots, \chi_{g_{N}}\right): \mathcal{T}(g, m) \rightarrow \mathbb{R}^{N}
$$

is a real analytic embedding. The number $N$ can be $N(g, m)$ in (1.1).

2.3.2. Canonical generators of type $(0,3)$ and $(1,1)$. In our paper we employ trace functions but not absolute values of trace functions in order to extend them to parameters for $S L(2, \mathbb{C})$ representation space of a surface group. For the results of this subsection we refer to [11, [2] or [18, §33]. Let $A \in S L(2, \mathbb{R})$. By $\operatorname{sgn} A$ we mean the sign of $\operatorname{tr} A$ and we write $\operatorname{sgn}\left(A_{1}, \ldots, A_{n}\right)$ for $\left(\operatorname{sgn} A_{1}, \ldots, \operatorname{sgn} A_{n}\right)$. Let $(A, B, C)(A B C=1)$ be a generator system of type $(0,3)$. So the axes of $A, B$ and $C$ correspond to the boundary components of $\mathbb{H} / G$. In this case

$$
\operatorname{sgn}(A, B, C)=(-,-,-),(+,+,-),(+,-,+) \text { or }(-,+,+) .
$$

If $(A, B, C)([A, B] C=1)$ is a generator system of type $(1,1)$ such that the axes of $A$ and $B$ project to simple closed geodesics in $\mathbb{H} / G$ with intersection number one and the axis of $[A, B]$ corresponds to the boundary curve, then

$$
\operatorname{sgn}(A, B, A B)=(+,+,+),(+,-,-),(-,+,-) \text { or }(-,-,+) \text { and } \operatorname{tr}[A, B]<0 .
$$

For the following theorems, see, for example, [18] and [6].

Theorem 2.2. Let $(A, B, C)(A B C=1)$ be a generator system of type $(0,3)$ with $\operatorname{tr} A<0$ and $\operatorname{tr} B<0$. Then $\operatorname{tr} C<0$. The mapping of $\mathcal{T}(0,3)$ into $\mathbb{R}_{>0}^{3}$ defined by

$$
(G,(A, B, C)) \mapsto(-\operatorname{tr} A,-\operatorname{tr} B,-\operatorname{tr} C)
$$

is a real analytic embedding. 
Theorem 2.3. Let $(A, B, C)\left(A B A^{-1} B^{-1} C=1\right)$ be a generator system of type $(1,1)$ with $\operatorname{tr} A>0$ and $\operatorname{tr} B>0$. Then $\operatorname{tr} A B>0$. The mapping of $\mathcal{T}(1,1)$ into $\mathbb{R}_{>0}^{3}$ defined by

$$
(G,(A, B, C)) \mapsto(\operatorname{tr} A, \operatorname{tr} B, \operatorname{tr} A B)
$$

is a real analytic embedding.

In other words, if $(g, m)=(0,3)$ or $(1,1)$, for each $X=(G,(A, B, C))$ in $\mathcal{T}(g, m)$, $\operatorname{tr} A, \operatorname{tr} B$ and $\operatorname{tr} A B$ recover $X$ (up to conjugacy).

Remark. If we are allowed to use as many trace functions as we like, we can prove Theorem 2.1 without difficulty by using Theorems 2.2 and 2.3 and Klein's combination theorem.

Theorem 2.4. Let $(A, B, C, D)(A B C D=1)$ be a generator system of type $(0,4)$ with $\operatorname{tr} A<0, \operatorname{tr} B<0, \operatorname{tr} C<0$ and $\operatorname{tr} D<0$. Then $\operatorname{tr} B C<0, \operatorname{tr} C A<0$ and $\operatorname{tr} A B<0$. The mapping of $\mathcal{T}(0,4)$ into $\mathbb{R}_{>0}^{7}$ defined by

$$
\begin{aligned}
& (G,(A, B, C, D)) \\
& \quad \mapsto \quad(a, b, c, d, x, y, z)=(-\operatorname{tr} A,-\operatorname{tr} B,-\operatorname{tr} C,-\operatorname{tr} D,-\operatorname{tr} B C,-\operatorname{tr} C A,-\operatorname{tr} A B)
\end{aligned}
$$

is a real analytic embedding and the image is contained in the zero locus of the polynomial (I5).

\section{Parametrization of the Teichmüller space of type $(1,2)$}

3.1. Trace identities. Let $\mathcal{S}=(A, B, C, D)\left(A B A^{-1} B^{-1} C D=1\right)$ be a generator system of a Fuchsian group of type $(1,2)$ such that

$$
a=\operatorname{tr} A>0, b=\operatorname{tr} B>0, c=-\operatorname{tr} C>0, d=-\operatorname{tr} D>0 .
$$

Our trace identity is suggested by the one for systems of type $(2,0)$, which will be given in Section 5. We use the following seven parameters which are all positive by (2.3) and (2.4):

$$
\begin{array}{ll}
c=-\operatorname{tr} C, & d=-\operatorname{tr} D, \quad u=\operatorname{tr} C A B A^{-1}, \quad v=\operatorname{tr} C A B^{2}, \\
w=\operatorname{tr} C A B, & z=\operatorname{tr} A B, \quad k=-\operatorname{tr} C D=-\operatorname{tr}[A, B] .
\end{array}
$$

We first remark that the following results remain the same as long as we consider positive trace functions. Let

$$
S=\sqrt{u v z-u^{2}-v^{2}-z^{2}}=\sqrt{-\operatorname{tr}\left[A B^{-1} A^{-1} C^{-1}, C A B^{2}\right]-2} .
$$

Then by (I3)

$$
\begin{aligned}
-S^{2}-2= & \operatorname{tr}\left(A B^{-1} A^{-1} C^{-1}\right)\left(C A B^{2}\right)\left(C A B A^{-1}\right)\left(B^{-2} A^{-1} C^{-1}\right) \\
= & \operatorname{tr}(A B C)\left(A B A^{-1} B^{-1}\right)\left(B^{-1} A^{-1} C^{-1}\right) \\
= & w \operatorname{tr}\left(A B A^{-1} B^{-1}\right)\left(B^{-1} A^{-1} C^{-1}\right)+(-k) \operatorname{tr}(A B C)\left(B^{-1} A^{-1} C^{-1}\right) \\
& +w \operatorname{tr}(A B C)\left(A B A^{-1} B^{-1}\right)-w^{2}(-k) \\
& -\operatorname{tr}(A B C)\left(B^{-1} A^{-1} C^{-1}\right)\left(A B A^{-1} B^{-1}\right) .
\end{aligned}
$$

We compute four traces in (3.3) by using (I1)-(I4). Let $x=\operatorname{tr} C B A$.

$$
\begin{aligned}
\operatorname{tr} A B A^{-1} B^{-2} A^{-1} C^{-1} & =\operatorname{tr}(C A B)(B A)\left(B^{-1} A^{-1}\right) \\
& =w(-k)-z c+z \operatorname{tr}(C A B)(B A)-w z z-x \\
& =-w k-z c+z\left(w z-\operatorname{tr} A B A^{-1} B^{-1} C\right)-z^{2} w-x \\
& =-w k-c z+d z-x \because A B A^{-1} B^{-1} C=D^{-1},
\end{aligned}
$$




$$
\begin{gathered}
\operatorname{tr}(A B C)\left(B^{-1} A^{-1}\right) C^{-1}=w^{2}+z^{2}+c^{2}+w z c-2, \\
\operatorname{tr}(A B C)\left(A B A^{-1} B^{-1}\right)=(-k) w-\operatorname{tr}(A B C)^{-1}\left(A B A^{-1} B^{-1}\right)=-k w-x .
\end{gathered}
$$

To compute the last trace in (3.3) we need

$$
\begin{aligned}
\operatorname{tr}(C A B)\left(C^{-1}\right)\left(A^{-1} B^{-1}\right) & =w x-c \operatorname{tr} C A B A^{-1} B^{-1}+z^{2}+w c z-\operatorname{tr}[A, B] \\
& =w x+c d+z^{2}+c z w+k .
\end{aligned}
$$

Then

$$
\begin{aligned}
& \operatorname{tr}\left(A B C B^{-1} A^{-1} C^{-1}\right)\left(A B A^{-1} B^{-1}\right) \\
&=\left(w^{2}+z^{2}+c^{2}+w z c-2\right)(-k) \\
&-\operatorname{tr}\left(A B C B^{-1} A^{-1} C^{-1}\right)^{-1}\left(A B A^{-1} B^{-1}\right) \quad \because(3.5) \\
&=-k\left(w^{2}+z^{2}+c^{2}+w z c-2\right)-\operatorname{tr}(C A B)\left(C^{-1}\right)\left(A^{-1} B^{-1}\right) \\
&=-k\left(w^{2}+z^{2}+c^{2}+w z c-1\right)-c z w-z^{2}-c d-w x .
\end{aligned}
$$

Substituting (3.4)-(3.7) into (3.3) we obtain

$$
S^{2}+k-k w^{2}+(c+d) z w+z^{2}+c d+2-w(c z+x)=0 .
$$

Since $(A B)\left(A^{-1} B^{-1}\right) C D=1$, and $(-z,-z, c, d, c z+x,-w, k)$ equals

$$
\left(-\operatorname{tr} A B,-\operatorname{tr} A^{-1} B^{-1},-\operatorname{tr} C,-\operatorname{tr} D,-\operatorname{tr} A^{-1} B^{-1} C,-\operatorname{tr} A B C,-\operatorname{tr} A B A^{-1} B^{-1}\right),
$$

substituting this into (I5) we obtain

$$
\begin{aligned}
& (c z+x)^{2}+(w k-c z-d z)(c z+x)+w^{2}+k^{2}-(c+d) z w+\left(c d+z^{2}\right) k \\
& +2 z^{2}+c^{2}+d^{2}+z^{2} c d-4=0 .
\end{aligned}
$$

Eliminating $c z+x$ from (3.8) and (3.9) we obtain

$$
\begin{aligned}
& w^{4}+(c+d) z w^{3}+\left(c^{2}+d^{2}-2 k-k S^{2}+2 z^{2}+c d z^{2}-4\right) w^{2} \\
& \quad+(c+d)\left(c d+k+S^{2}+z^{2}+2\right) z w+\left(2+c d+k+S^{2}+z^{2}\right)^{2}=0 .
\end{aligned}
$$

The left-hand side of this equation equals

$$
\begin{aligned}
& \left(w^{2}+\frac{c+d}{2} z w+k+S^{2}+z^{2}+c d+2\right. \\
& \left.-w \sqrt{(k+2)\left(S^{2}+4\right)+\left(\frac{c-d}{2}\right)^{2}\left(z^{2}-4\right)}\right) \\
& \quad+\left(w^{2}+\frac{c+d}{2} z w+k+S^{2}+z^{2}+c d+2\right. \\
& \left.+w \sqrt{(k+2)\left(S^{2}+4\right)+\left(\frac{c-d}{2}\right)^{2}\left(z^{2}-4\right)}\right) .
\end{aligned}
$$

Since the variables are all positive, we obtain

$$
w^{2}+\frac{c+d}{2} z w+k+S^{2}+z^{2}+c d+2-w \sqrt{(k+2)\left(S^{2}+4\right)+\left(\frac{c-d}{2}\right)^{2}\left(z^{2}-4\right)}=0 .
$$

We denote the left-hand side of (3.11) by $G_{12}(u, v, z, w, c, d, k)$. 
3.2. Parametrization of $\mathcal{T}(1,2)$. We show the seven traces in (3.2) give a global parametrization of the Teichmüller space $\mathcal{T}(1,2)$. Recall that $c=-\operatorname{tr} C, d=-\operatorname{tr} D$, $z=\operatorname{tr} A B, w=\operatorname{tr} A B C$. Let

$$
a=\operatorname{tr} A, \quad b=\operatorname{tr} B, \quad x_{1}=\operatorname{tr} A C, \quad x_{2}=\operatorname{tr} B C .
$$

By using (I1)-(I3) again, we compute some traces.

(1) $v=\operatorname{tr} C A B^{2}=w b-x_{1}$.

(2) Since $-d=\operatorname{tr} D=\operatorname{tr}(A B)(B A)^{-1} C=\operatorname{tr}(B A)^{-1}(C A B)$,

$$
\begin{aligned}
-d & =z w-\operatorname{tr} B(B A C A)=z w-b \operatorname{tr}(B A)(C A)+\operatorname{tr} A C A \\
& =z w-b\left(z x_{1}-\operatorname{tr} A^{-1} B^{-1} C A\right)+\left(a x_{1}-(-c)\right) \\
& =z w-b z x_{1}+b\left(b(-c)-x_{2}\right)+a x_{1}+c \\
& =z w+c+(a-b z) x_{1}-b c^{2}-b x_{2} .
\end{aligned}
$$

(3) Since $u=\operatorname{tr} C(A B) A^{-1}$,

$$
\begin{aligned}
u & =(-c) b+z \operatorname{tr} C A^{-1}+a w-(-c) z a-\operatorname{tr} C A^{-1}(A B) \\
& =-b c+z\left((-c) a-x_{1}\right)+a w+a c z-x_{2}=-b c+a w-z x_{1}-x_{2} .
\end{aligned}
$$

Using (2) and (3) eliminate $x_{2}$ and substitute $x_{1}=w b-v$ from (1) into the result. Then

$$
a=\frac{c+d+z w+b u}{v} .
$$

Substitute this to $k=-\operatorname{tr}[A, B]=a b z-a^{2}-b^{2}-z^{2}+2$, then we have

$$
\left(S^{2}+z^{2}\right) b^{2}-(2 u-z v)(c+d+z w) b-(c+d+z w)^{2}-\left(z^{2}+k-2\right) v^{2}=0,
$$

where $S^{2}=u v z-u^{2}-v^{2}-z^{2}$ as before. Since $b$ is positive

$$
b=\frac{(2 u-z v)(c+d+z w)+\sqrt{\Delta}}{2\left(S^{2}+z^{2}\right)},
$$

where

$$
\begin{aligned}
\Delta= & (2 u-z v)^{2}(c+d+z w)^{2}+4\left(S^{2}+z^{2}\right)\left[(c+d+z w)^{2}+\left(z^{2}+k-2\right) v^{2}\right] \\
= & 4 v^{2}\left((k+2)\left(S^{2}+4\right)+\left(\frac{c-d}{2}\right)^{2}\left(z^{2}-4\right)\right)+v^{2} w^{2}\left(z^{2}-4\right)^{2} \\
& +4\left(z^{2}-4\right) v^{2}\left(w^{2}+\frac{c+d}{2} z w+k+S^{2}+z^{2}+c d+2\right) \\
= & 4 v^{2}\left(\frac{w^{2}+\frac{c+d}{2} z w+k+S^{2}+z^{2}+c d+2}{w}\right)^{2}+v^{2} w^{2}\left(z^{2}-4\right)^{2} \\
& +4\left(z^{2}-4\right) v^{2}\left(w^{2}+\frac{c+d}{2} z w+k+S^{2}+z^{2}+c d+2\right) \quad \because(3.11) \\
= & \frac{v^{2}}{w^{2}}\left(\left(z^{2}-4\right) w^{2}+2\left(w^{2}+\frac{c+d}{2} z w+k+S^{2}+z^{2}+c d+2\right)\right)^{2} .
\end{aligned}
$$

Hence we obtain from this and (3.13),

$$
\begin{aligned}
& a=\frac{w(u z-v)(c+d+z w)+u\left(2+c d+k+S^{2}+z^{2}-w^{2}\right)}{w\left(S^{2}+z^{2}\right)}, \\
& b=\frac{u w(c+d+z w)+v\left(2+c d+k+S^{2}+z^{2}-w^{2}\right)}{w\left(S^{2}+z^{2}\right)} .
\end{aligned}
$$


Then from (1) and (3),

$$
\begin{aligned}
& x_{1}=w b-v=\frac{(2+c d+k) v+(u z-v) w^{2}+(c+d) u w}{S^{2}+z^{2}}, \\
& x_{2}=-z x_{1}+a w-b c-u .
\end{aligned}
$$

Let $\mathcal{S}_{0}=(A, B, C)$. Since $[A, B] C D=1, G_{\mathcal{S}}=G_{\mathcal{S}_{0}}$. We showed that all traces in the set $T_{\mathcal{S}_{0}}$ (see (2.3)) are rational functions in $(u, v, z, w, c, d, k)$ with integer coefficients, and hence so are all traces of elements in $G_{\mathcal{S}}=\langle A, B, C, D\rangle$. By using Theorem 2.1] we can conclude the following.

Theorem 3.1. For a generator system $\mathcal{S}=(A, B, C, D)$ of type $(1,2)$ define the tuple of trace functions $(u, v, z, w, c, d, k)$ by (3.2). Then the mapping $\Phi_{12}\left(G_{\mathcal{S}}, \mathcal{S}\right)=$ $(u, v, z, w, c, d, k)$ embeds the Teichmüller space $\mathcal{T}(1,2)$ into $\mathbb{R}_{>0}^{7}$. The image of $\Phi_{12}$ is contained in the zero locus of $G_{12}(u, v, z, w, c, d, k)$ in (3.11).

The polynomial in (3.10) is a quadratic polynomial in $d$ and the coefficient of $d^{2}$ is $c^{2}+w^{2}+c w z$ and the coefficient of $d$ is

$$
w z\left(c^{2}+w^{2}+c w z\right)+(2 c+z w)\left(2+k+S^{2}+z^{2}\right) .
$$

They are positive. So $d$ is the unique positive solution of (3.10) and can be omitted. The Teichmüller space $\mathcal{T}(1,2)$ is parametrized by $\operatorname{six}(=\operatorname{dim} \mathcal{T}(1,2))$ parameters $(u, v, z, w, c, k)$.

If $G=\left\langle A, B, C_{1}, C_{2}\right\rangle\left(C_{1}=C, C_{2}=D\right)$, the mapping class group $\mathcal{M} C_{12}$ of $\mathbb{H} / G$ is isomorphic to the group of the outer automorphisms $\alpha$ of $G$ satisfying

$$
\alpha\left(C_{j}\right)=l_{j}^{-1} C_{\sigma(j)} l_{j} \text { for } j=1,2,
$$

for some $l_{j} \in G$ and a permutation $\sigma$ of $\{1,2\}$. (Nielsen's theorem, see [18, §11].) Since $\alpha$ is determined by the images of $A, B, C$ and $D$, we obtain the following theorem.

Theorem 3.2. The mapping class group $\mathcal{M} C_{12}$ acts on the image of $\mathcal{T}(1,2)$ under $\Phi_{12}$ by a group of rational transformations.

For example, the outer automorphism $\omega$ of $G$ defined by

$$
(A, B, C, D) \mapsto(A, B A, C, D)
$$

acts on the parameter space by sending $(u, v, z, w, c, d, k)$ to

$$
\left(\operatorname{tr} C A(B A) A^{-1}, \operatorname{tr} C A(B A)^{2}, \operatorname{tr} A(B A), \operatorname{tr} C A(B A),-\operatorname{tr} C,-\operatorname{tr} D,-\operatorname{tr} C D\right) .
$$

(1)

$$
\begin{aligned}
\operatorname{tr} C A(B A) & =x_{1} z-\operatorname{tr}(C A)(B A)^{-1}=z x_{1}-\operatorname{tr} C B^{-1} \\
& =x_{1} z+b c+x_{2} .
\end{aligned}
$$

$(2)$

$$
\begin{aligned}
\operatorname{tr} C A(B A)^{2} & =(\operatorname{tr} C A(B A)) z-x_{1}=\left(x_{1} z+b c+x_{2}\right) z-x_{1} \quad \because(1) \\
& =\left(z^{2}-1\right) x_{1}+\left(x_{2}+b c\right) z .
\end{aligned}
$$

(3) $\operatorname{tr} A(B A)=a z-\operatorname{tr} A^{-1} B A=a z-b$.

Hence $\omega$ induces

$$
\omega_{*}(u, v, z, w, c, d, k)=\left(w,\left(z^{2}-1\right) x_{1}+\left(x_{2}+b c\right) z, a z-b, x_{1} z+b c+x_{2}, c, d, k\right) .
$$


By (3.14) and (3.15) this is a rational transformation in $(u, v, z, w, c, d, k)$. This is a very simple case. For a general automorphism, the rational transformation has a huge expression.

\section{Teichmüller SPACE OF ClOSED SURFACE OF Genus two}

Let $\mathcal{S}=(A, B, C, D)([A, B][C, D]=1)$ be a generator system of type $(2,0)$ such that

$$
\operatorname{tr} A>0, \quad \operatorname{tr} B>0, \quad \operatorname{tr} C>0, \quad \operatorname{tr} D>0 .
$$

In order to treat positive values, we use the following seven parameters:

$$
\begin{array}{ll}
c=\operatorname{tr} C, & d=\operatorname{tr} D, \quad u=-\operatorname{tr} C A B A^{-1}, \quad v=-\operatorname{tr} C A B^{2}, \\
w=-\operatorname{tr} C A B, & z=\operatorname{tr} A B, \quad t=\operatorname{tr} C D .
\end{array}
$$

$\mathcal{S}_{1}=\left(A, B, C, D C^{-1} D^{-1}\right)$ is a generator system of type $(1,2)$. The results in Section 3 show that traces of elements of $G_{\mathcal{S}_{1}}$ are rational functions with variables $u$, $v, z, w, c=\operatorname{tr} C=\operatorname{tr} D C^{-1} D^{-1}$ and $K^{2}=c d t-c^{2}-d^{2}-t^{2}=-\operatorname{tr} C\left(D C^{-1} D^{-1}\right)-2$, which satisfy $G_{12}\left(u, v, z, w, c, c, K^{2}+2\right)=0$ or

$$
w^{2}+c z w+K^{2}+S^{2}+z^{2}+c^{2}+4-w \sqrt{\left(K^{2}+4\right)\left(S^{2}+4\right)}=0,
$$

where $S^{2}=u v z-u^{2}-v^{2}-z^{2}$. In particular, $\operatorname{tr} A, \operatorname{tr} B, \operatorname{tr} C, \operatorname{tr} A B, \operatorname{tr} A C, \operatorname{tr} B C$, $\operatorname{tr} A B C$ are rational functions in $(u, v, z, w, c, d, t)$. Other members of $T_{\mathcal{S}}$ are $d=$ $\operatorname{tr} D, \operatorname{tr} A D, \operatorname{tr} B D, t=\operatorname{tr} C D, \operatorname{tr} A B D, \operatorname{tr} A C D, \operatorname{tr} B C D$ and $\operatorname{tr} A B C D$. Let $X=A$, $B$ or $A B$. Then $\operatorname{tr} X, \operatorname{tr} X C, \operatorname{tr} X D C^{-1} D^{-1}$ and $\operatorname{tr} X C\left(D C^{-1} D^{-1}\right)$ belong to $T_{\mathcal{S}_{1}}$. Let $x=\operatorname{tr} X$. Then

$$
\begin{aligned}
\operatorname{tr} X D\left(C^{-1} D^{-1}\right)= & (\operatorname{tr} X D) t-\operatorname{tr}(X D)(D C)=(\operatorname{tr} X D) t-\operatorname{tr} D(D C X) \\
= & (\operatorname{tr} X D) t-(d \operatorname{tr} X D C-\operatorname{tr} X C) \\
= & (\operatorname{tr} X D) t+\operatorname{tr} X C-d(x t+d \operatorname{tr} X C+c \operatorname{tr} X D-x d c-\operatorname{tr} X C D) \\
= & (t-c d) \operatorname{tr} X D+d \operatorname{tr} X C D+\left(1-d^{2}\right) \operatorname{tr} X C-d x t+x d^{2} c \\
\operatorname{tr} X C D\left(C^{-1} D^{-1}\right)= & t(\operatorname{tr} X C D)-\operatorname{tr} X C D^{2} C \\
= & t(\operatorname{tr} X C D)-\left(d(\operatorname{tr}(X C)(D C))-\operatorname{tr} X C^{2}\right) \\
= & t(\operatorname{tr} X C D)-d\left((\operatorname{tr} X C) t-\operatorname{tr} X D^{-1}\right)+(c(\operatorname{tr} X C)-x) \\
= & t(\operatorname{tr} X C D)+d(x d-\operatorname{tr} X D)+(c-d t) \operatorname{tr} X C-x \\
= & -d \operatorname{tr} X D+t \operatorname{tr} X C D+(c-d t) \operatorname{tr} X C+\left(d^{2}-1\right) x .
\end{aligned}
$$

Since $\operatorname{tr}[C, D]<-2$,

$$
\left|\begin{array}{cc}
t-c d & d \\
-d & t
\end{array}\right|=\operatorname{tr}[C, D]-c^{2}+2<0 .
$$

By these equations, $\operatorname{tr} X D$ and $\operatorname{tr} X C D$ are expressed by rational functions in $(u, v, z, w, c, d, t)$. Therefore all traces in $T_{\mathcal{S}}$ and hence all traces of elements of $G_{\mathcal{S}}$ are rational functions in these seven parameters. As in the case of type $(1,2)$ we obtain the following theorem.

Theorem 4.1. For a generator system $\mathcal{S}=(A, B, C, D)$ of type $(2,0)$ define $u, v$, $z, w, c, d$ and $t$ by (4.1). Then the mapping $\Phi_{20}\left(G_{\mathcal{S}}, \mathcal{S}\right)=(u, v, z, w, c, d, t)$ embeds the Teichmüller space $\mathcal{T}(2,0)$ into $\mathbb{R}_{>0}^{7}$. The image of $\Phi_{20}$ is contained in the zero locus of the left-hand side in (4.2) with

$$
S=\sqrt{u v z-u^{2}-v^{2}-z^{2}} \quad \text { and } \quad K=\sqrt{c d t-c^{2}-d^{2}-t^{2}} .
$$


Moreover, the mapping class group $\mathcal{M C}_{20}$ of the closed surface of genus two acts on the image of $\mathcal{T}(2,0)$ under $\Phi_{20}$ by a group of rational transformations.

\section{A tRace identity FOR GROUPS OF TyPe $(2,0)$}

The fact that all closed Riemann surfaces of genus two are hyperelliptic yields a canonical identification of $\mathcal{T}(2,0)$ with the Teichmüller space of the sphere with six cone points of angle $\pi$ (or of signature $(0 ; 2,2,2,2,2,2 ; 0 ; 0)$ ); see [18). By using a triangulation of the sphere with six cone points of angle $\pi$, we can give global coordinates for $\mathcal{T}(2,0)$ by seven geodesic length functions (see, for example, [8]). In this section we deduce the trace identity (4.2) in a different way. This identity hinted to us the identity (3.11).

A Fuchsian group $G$ of type $(2,0)$ is a subgroup with index 2 of a Fuchsian group $\Gamma$ with signature $(0 ; 2,2,2,2,2,2 ; 0 ; 0)$, having a generator system $\left(e_{1}, e_{2}, e_{3}, e_{4}\right.$, $\left.e_{5}, e_{6}\right)$ such that $e_{1} e_{2} e_{3} e_{4} e_{5} e_{6}=e_{1}^{2}=e_{2}^{2}=\cdots=e_{6}^{2}=1$. By setting

$$
A=e_{1} e_{3}, B=e_{3} e_{2}, C=e_{4} e_{6}, D=e_{6} e_{5},
$$

we obtain a canonical generator system of $G$ with $[A, B][C, D]=1$.

Since $\left(B^{-1}, B A B^{-1}, C, D\right)$ is also a canonical generator system, the argument in Section 4 shows that $\operatorname{tr} A, \operatorname{tr} B, \operatorname{tr} A B,-\operatorname{tr} B^{-1} C D,-\operatorname{tr} B^{-1} C D C^{-1},-\operatorname{tr} B^{-1} C D^{2}$ and $\operatorname{tr} C D$ give global coordinates for $\mathcal{T}(2,0)$.

Let $E_{k}$ be a matrix in $S L(2, \mathbb{R})$ representing $e_{k}(k=1,2, \ldots, 6)$ with $E_{1} \cdots E_{6}=1$. Since $E_{k}$ is elliptic of order $2, \operatorname{tr} E_{k}=0$ and $E_{k}^{-1}=-E_{k}$. Let $Q=E_{1} E_{2} E_{3}$. Since $A B A^{-1} B^{-1}=-\left(E_{1} E_{2} E_{3}\right)^{2}=-Q^{2}$, by setting $a=\operatorname{tr} A, b=\operatorname{tr} B, z=$ $\operatorname{tr} A B=-\operatorname{tr} E_{1} E_{2}$ and $K=\sqrt{a b z-a^{2}-b^{2}-z^{2}}$ we have $(\operatorname{tr} Q)^{2}=K^{2}+4$. Next let $R=E_{1} E_{5} E_{4}$. By the trace identity (I3) in Subsection 2.2 and that $\operatorname{tr} E_{k}=0$, we have $\operatorname{tr} R=-\operatorname{tr} E_{1} E_{4} E_{5}$. Since

$$
\begin{aligned}
& \left(B^{-1} C D C^{-1}\right)(C D)\left(B^{-1} C D C^{-1}\right)^{-1}(C D)^{-1} \\
& \quad=-E_{2} E_{3} E_{4} E_{5} E_{6} E_{5} E_{4} E_{6} E_{5} E_{4} E_{3} E_{2} E_{5} E_{4}=-R^{2},
\end{aligned}
$$

by setting $t=\operatorname{tr} C D=-\operatorname{tr} E_{4} E_{5}$,

$$
\begin{aligned}
& u=-\operatorname{tr} B^{-1} C D C^{-1}=\operatorname{tr} E_{2} E_{3} E_{4} E_{5} E_{6} E_{4}=-\operatorname{tr} E_{1} E_{4}, \\
& v=-\operatorname{tr} B^{-1} C D^{2}=\operatorname{tr} E_{2} E_{3} E_{4} E_{5} E_{6} E_{5}=-\operatorname{tr} E_{1} E_{5}
\end{aligned}
$$

and $S=\sqrt{u v t-u^{2}-v^{2}-t^{2}}$, we have $(\operatorname{tr} R)^{2}=S^{2}+4$. Let

$$
w=-\operatorname{tr} B^{-1} C D=\operatorname{tr} E_{2} E_{3} E_{4} E_{5}=\operatorname{tr} E_{6} E_{1} .
$$

Since $\left(E_{6} E_{1}, E_{2} E_{3}, E_{4} E_{5}\right)$ is a system of type $(0,3)$, if $b=\operatorname{tr} B=\operatorname{tr} E_{2} E_{3}>0$ and $-t=-\operatorname{tr} C D=\operatorname{tr} E_{4} E_{5}<0$, then $w>0$. We have

$$
\begin{aligned}
& \left(E_{1} E_{2} E_{3}\right)\left(E_{4} E_{5} E_{1}\right)\left(E_{1} E_{2} E_{3}\right)^{-1}\left(E_{4} E_{5} E_{1}\right)^{-1} \\
& \quad=\left(E_{1} E_{2} E_{3} E_{4} E_{5} E_{6}\right)\left(E_{6} E_{1} E_{3} E_{2} E_{5} E_{4}\right) \\
& \quad=\left(-E_{6} E_{1}\right)\left(E_{3} E_{2}\right)\left(E_{5} E_{6}^{2} E_{4}\right) \\
& \quad=\left(B^{-1} C D\right)^{-1}\left(B D^{-1} C^{-1}\right) .
\end{aligned}
$$

Since the axes of $E_{1} E_{2} E_{3}$ and $E_{4} E_{5} E_{1}$ intersect, $\operatorname{tr}\left[E_{1} E_{2} E_{3}, E_{4} E_{5} E_{1}\right]<-2$, and this implies

$$
\operatorname{tr}\left(E_{1} E_{2} E_{3}\right) \operatorname{tr}\left(E_{4} E_{5} E_{1}\right) \operatorname{tr}\left(E_{1} E_{2} E_{3} E_{4} E_{5} E_{1}\right)=\operatorname{tr}\left(E_{1} E_{2} E_{3}\right) \operatorname{tr}\left(E_{4} E_{5} E_{1}\right)(-w)>0
$$


(see [11, Section 2]). Hence we have from (5.1),

$$
\left(K^{2}+4\right)+\left(S^{2}+4\right)+w^{2}-w \sqrt{K^{2}+4} \sqrt{S^{2}+4}-2=\operatorname{tr}\left(B^{-1} C D\right)^{-1} B D^{-1} C^{-1} .
$$

Since

$$
\operatorname{tr} B\left(B^{-1} C D\right) B^{-1}\left(D^{-1} C^{-1} B\right)=\operatorname{tr} C D B^{-1} D^{-1} C^{-1} B=\operatorname{tr}\left(B^{-1} C D\right)\left(B D^{-1} C^{-1}\right),
$$

$$
b^{2}+w^{2}+t^{2}+b w t-2=\operatorname{tr}\left(B^{-1} C D\right)\left(B D^{-1} C^{-1}\right) .
$$

The trace identity (I2) yields

$$
\operatorname{tr}\left(B^{-1} C D\right)^{-1}\left(B D^{-1} C^{-1}\right)+\operatorname{tr}\left(B^{-1} C D\right)\left(B D^{-1} C^{-1}\right)=w^{2} .
$$

By adding (5.2) and (5.3) we obtain the following identity

$$
\left(K^{2}+4\right)+\left(S^{2}+4\right)-w \sqrt{\left(K^{2}+4\right)\left(S^{2}+4\right)}+w^{2}+t^{2}+b^{2}+w t b-4=0 .
$$

\section{An exAmple}

We conclude this paper by an example of a Fuchsian group of type $(2,0)$ such that traces of its elements are all integers. Let $\mathcal{S}=(A, B, C, D)$, where

$$
\begin{array}{ll}
A=\left(\begin{array}{cc}
\frac{-1+2 \sqrt{2}}{\sqrt{2}} & \frac{\sqrt{5}(1+\sqrt{2})}{\sqrt{2}} \\
\frac{\sqrt{5}(-1+\sqrt{2})}{\sqrt{2}} & \frac{1+2 \sqrt{2}}{\sqrt{2}}
\end{array}\right), \quad B=\left(\begin{array}{cc}
\frac{-1+2 \sqrt{2}}{\sqrt{2}} & -\frac{\sqrt{5}(1+\sqrt{2})}{\sqrt{2}} \\
\frac{\sqrt{5}(1-\sqrt{2})}{\sqrt{2}} & \frac{1+2 \sqrt{2}}{\sqrt{2}}
\end{array}\right), \\
C=\left(\begin{array}{cc}
\frac{1+2 \sqrt{2}}{\sqrt{2}} & \frac{\sqrt{5}(1-\sqrt{2})}{\sqrt{2}} \\
-\frac{\sqrt{5}(1+\sqrt{2})}{\sqrt{2}} & \frac{-1+2 \sqrt{2}}{\sqrt{2}}
\end{array}\right), & D=\left(\begin{array}{cc}
\frac{1+2 \sqrt{2}}{\sqrt{2}} & \frac{\sqrt{5}(-1+\sqrt{2})}{\sqrt{2}} \\
\frac{\sqrt{5}(1+\sqrt{2})}{\sqrt{2}} & \frac{-1+2 \sqrt{2}}{\sqrt{2}}
\end{array}\right) .
\end{array}
$$

Then $\mathcal{S}$ is a generator system of type $(2,0)$. Actually, the group $G_{1}=\langle A, B\rangle$ is a Fuchsian group of type $(1,1)$ such that the axis of $[A, B]$ is the unit circle, and $G_{2}=\langle C, D\rangle$ is the conjugate of $G_{1}$ by the reflection in the unit circle. Since all traces in $T_{\mathcal{S}}$ are integers,

$$
\begin{array}{r}
\operatorname{tr}\{A, B, C, D, A B, A C, A D, B C, B D, C D, A B C, A B D, A C D, B C D, A B C D\} \\
=\{4,4,4,4,4,-8,22,22,-8,4,-16,24,-16,24,-38\},
\end{array}
$$

all traces of the elements in $G_{\mathcal{S}}$ are integers.

\section{REFERENCES}

[1] Alan F. Beardon, The geometry of discrete groups, Graduate Texts in Mathematics, vol. 91, Springer-Verlag, New York, 1983. MR698777 (85d:22026)

[2] J. Gilman and B. Maskit, An algorithm for 2-generator Fuchsian groups, Michigan Math. J. 38 (1991), no. 1, 13-32, DOI 10.1307/mmj/1029004258. MR1091506 (92f:30062)

[3] Ursula Hamenstädt, Length functions and parameterizations of Teichmüller space for surfaces with cusps, Ann. Acad. Sci. Fenn. Math. 28 (2003), no. 1, 75-88. MR1976831 (2004e:30070)

[4] Linda Keen, On Fricke moduli, Advances in the Theory of Riemann Surfaces (Proc. Conf., Stony Brook, N.Y., 1969), Ann. of Math. Studies, No. 66, Princeton Univ. Press, Princeton, N.J., 1971, pp. 205-224. MR0288252 (44 \#5450)

[5] Irwin Kra, On lifting Kleinian groups to SL(2, C), Differential geometry and complex analysis, Springer, Berlin, 1985, pp. 181-193. MR780044 (86h:30078)

[6] Feng Luo, Geodesic length functions and Teichmüller spaces, J. Differential Geom. 48 (1998), no. 2, 275-317. MR1630186 (99e:32031)

[7] Colin Maclachlan and Alan W. Reid, The arithmetic of hyperbolic 3-manifolds, Graduate Texts in Mathematics, vol. 219, Springer-Verlag, New York, 2003. MR1937957 (2004i:57021) 
[8] Toshihiro Nakanishi and Marjatta Näätänen, Parametrization of Teichmüller space by length parameters, Analysis and topology, World Sci. Publ., River Edge, NJ, 1998, pp. 541-560. MR.1667832 (99m:30092)

[9] Yoshihide Okumura, On the global real analytic coordinates for Teichmüller spaces, J. Math. Soc. Japan 42 (1990), no. 1, 91-101, DOI 10.2969/jmsj/04210091. MR.1027542 (90k:32071)

[10] Yoshihide Okumura, Global real analytic length parameters for Teichmüller spaces, Hiroshima Math. J. 26 (1996), no. 1, 165-179. MR1380431 (97b:32021)

[11] Norman Purzitsky, Two-generator discrete free products, Math. Z. 126 (1972), 209-223. MR0346070 (49 \#10796)

[12] Paul Schmutz, Die Parametrisierung des Teichmüllerraumes durch geodätische Längenfunktionen, Comment. Math. Helv. 68 (1993), no. 2, 278-288, DOI 10.1007/BF02565819 (German). MR1214232(94g:32028)

[13] Mika Seppälä and Tuomas Sorvali, On geometric parametrization of Teichmüller spaces, Ann. Acad. Sci. Fenn. Ser. A I Math. 10 (1985), 515-526. MR802516 (87e:32032)

[14] Mika Seppälä and Tuomas Sorvali, Parametrization of Teichmüller spaces by geodesic length functions, Holomorphic functions and moduli, Vol. II (Berkeley, CA, 1986), Math. Sci. Res. Inst. Publ., vol. 11, Springer, New York, 1988, pp. 267-284, DOI 10.1007/978-1-4613-96116_18. MR.955845 (89m:32040)

[15] Mika Seppälä and Tuomas Sorvali, Parametrization of Möbius groups acting in a disk, Comment. Math. Helv. 61 (1986), no. 1, 149-160, DOI 10.1007/BF02621907. MR847525 (87i:20089)

[16] Mika Seppälä and Tuomas Sorvali, Geometry of Riemann surfaces and Teichmüller spaces, North-Holland Mathematics Studies, vol. 169, North-Holland Publishing Co., Amsterdam, 1992. MR 1202043 (94b:32034)

[17] Scott A. Wolpert, Geodesic length functions and the Nielsen problem, J. Differential Geom. 25 (1987), no. 2, 275-296. MR880186 (88e:32032)

[18] Heiner Zieschang, Finite groups of mapping classes of surfaces, Lecture Notes in Mathematics, vol. 875, Springer-Verlag, Berlin, 1981. MR643627 (86g:57001)

Science Division, Center for General Education, Aichi Institute of Technology, 1247 Yachigusa, Yakusa, Toyota, 470-0392, JAPAN

E-mail address: gou@aitech.ac.jp

Department of Mathematics, Shimane University, Matsue, 690-8504, Japan

E-mail address: tosihiro@riko.shimane-u.ac.jp 